\title{
Distribution of Podoplanin-Expressing Cells in the Mouse Nervous Systems
}

\author{
Miwa Tomooka ${ }^{1}$, Chiaki Kaji ${ }^{1}$, Hiroshi Kojima ${ }^{1}$ and Yoshihiko Sawa ${ }^{2}$ \\ ${ }^{1}$ Department of Oral Growth \& Development, Fukuoka Dental College and ${ }^{2}$ Department of Morphological Biology, \\ Fukuoka Dental College
}

Received October 18, 2013; accepted November 26, 2013; published online December 25, 2013

\begin{abstract}
Podoplanin is a mucin-type glycoprotein which was first identified in podocytes. Recently, podoplanin has been successively reported as a marker for brain and peripheral nerve tumors, however, the distribution of podoplanin-expressing cells in normal nerves has not been fully investigated. This study aims to examine the podoplanin-expressing cell distribution in the mouse head and nervous systems. An immunohistochemical study showed that the podoplanin-positive areas in the mouse peripheral nerve and spinal cord are perineurial fibroblasts, satellite cells in the dorsal root ganglion, glia cells in the ventral and dorsal horns, and schwann cells in the ventral and dorsal roots; in the cranial meninges the dura mater, arachnoid, and pia mater; in the eye the optic nerve, retinal pigment epithelium, chorioidea, sclera, iris, lens epithelium, corneal epithelium, and conjunctival epithelium. In the mouse brain choroid plexus and ependyma were podoplanin-positive, and there were podoplaninexpressing brain parenchymal cells in the nuclei and cortex. The podoplanin-expressing cells were astrocyte marker GFAP-positive and there were no differences in the double positive cell distribution of several portions in the brain parenchyma except for the fornix. The results suggest that podoplanin may play a common role in nervous system support cells and eye constituents.
\end{abstract}

Key words: podoplanin, nervous system, glia cell, astrocyte

\section{Introduction}

Podoplanin, also termed Aggrus/gp36/E11 antigen, is a $38-\mathrm{kDa}$ (mouse) or $36-\mathrm{kDa}$ (human) mucin-type glycoprotein with a high content of sialic acid $[12,13]$ which was first identified in kidney glomerular epithelial cells, podocytes $[3,26]$. The Ebp1 is the only transcriptional factor which has been reported to promote tumorigenesis by the activation of the podoplanin gene [18]. In gliomas, increases in podoplanin are caused by loss of PTEN and activation of the PI3K-AKT-AP-1 signaling pathway, accompanied by epigenetic regulation of PDPN promoter activity. Silencing of the PDPN expression leads to reduced proliferation and migration of glioma cells [21]. Therefore, podoplanin has been considered as a candidate for target

Correspondence to: Yoshihiko Sawa, Fukuoka Dental College, Department of Morphological Biology, 2-15-1 Tamura, Sawara-ku, Fukuoka 814-0193, Japan. E-mail: ysawa@college.fdcnet.ac.jp protein in brain cancer treatment.

Podoplanin is commonly known as a useful marker for lymphatic endothelium and type I alveolar epithelium $[22,24,32]$. Podoplanin is also expressed in osteocytes and osteoblasts [31], choroid plexus [11], perineurium [20], mesothelial cells $[4,14]$, epidermal basal layer cells [24], tooth germ epithelial cells, salivary gland myoepithelium [1, 6-8, 20, 23], thymus type I epithelial cells, prostate myofibroblasts, follicular dendritic cells, and immature cells such as fetal germ cells and developing Sertoli cells [16, 25, 29, 33]. In nervous system tumors, expression of podoplanin in schwannomas, ependymal tumors, choroid plexus papillomas, meningiomas, astrocytic tumors, medulloblastomas, and hemangioblastomas have been reported $[9,10,19,27]$. Therefore, it is thought that glia cells commonly express podoplanin, however, the distribution of podoplanin-positive cells in the nervous system has not been fully investigated. This study aims to examine the expression of podoplanin in the nervous system.

(C) 2013 The Japan Society of Histochemistry and Cytochemistry 


\section{Materials and Methods}

\section{Animals}

Closed line ICR mice (Japan Clea Inc., Osaka, Japan) of gestational day 7 were purchased and one week-old male mice $(n=10)$ were used. Breeding and experiences were performed in a room with $100 \%$ controlled atmosphere which had passed an examination for bacteria in the Fukuoka Dental College Animal Center. Mice grew normally and lived healthily under conventional atmosphere conditions with normal feeding in cages and rooms in which lighting and temperature $\left(22^{\circ} \mathrm{C}\right)$ were completely controlled. The collection of the tissue was conducted after euthanasia by $2 \%(1 \mathrm{l} / \mathrm{min})$ isoflurane inhalation with an anaesthetic apparatus and cervical dislocation. The protocol for the animal use was reviewed and approved by the animal experiment committee of Fukuoka Dental College in accordance with the principles of the Helsinki Declaration.

\section{Immunohistochemistry}

Tissue was fixed in $100 \%$ methanol for $10 \mathrm{~min}$ at $-20^{\circ} \mathrm{C}$, and then immersed in $30 \%$ sucrose-PBS for $12 \mathrm{hr}$ at $4^{\circ} \mathrm{C}$ before freezing. Frozen $10 \mu \mathrm{m}$ sections were cut in a cryostat and fixed in $100 \%$ methanol for $5 \mathrm{~min}$ at $-20^{\circ} \mathrm{C}$. To make paraffin-embedded sections, perfusion fixation was performed through the heart with $500 \mathrm{ml}$ of fixative solution containing $4 \%$ formaldehyde-PBS after the euthanasia described above. The dissected tissue was additionally fixed by immersion in a $10 \%$ formalin-PBS solution for 8 $\mathrm{hr}$ at room temperature as fixing of the tissue for longer than $24 \mathrm{hr}$ leads to masking or destruction of the podoplanin antigen. The $5-\mu \mathrm{m}$ thick paraffin-embedded sections were cut using a rotary microtome, mounted on 3-aminopropyltriethoxysilane-coated slides, deparaffinized in changes of xylene, rehydrated in decreasing concentrations of ethanol, and rinsed with deionized distilled water for $10 \mathrm{~min}$. Slides were immersed in $10 \mathrm{mM}$ sodium citrate buffer $(\mathrm{pH}$ 6.0) and boiled in a microwave oven for $10 \mathrm{~min}$ to break formalin-formed protein cross-links masking the podoplanin antigen. After the boiling, the sections were rinsed by $10 \mathrm{mM}$ PBS and blocked with $0.1 \%$ goat serum. The immunostaining was performed using the first and second antibodies diluted into the blocking solution. All procedures were performed after washing with $10 \mathrm{mM}$ PBS for $30 \mathrm{~min}$ at room temperature. Sections were exposed by primary antibodies: $1 \mu \mathrm{g} / \mathrm{ml}$ of monoclonal hamster anti-mouse podoplanin (AngioBio Co., Del Mar, CA) and $1 \mu \mathrm{g} / \mathrm{ml}$ of polyclonal rabbit anti-mouse glial fibrillary acidic protein (GFAP, Abcam plc., Cambridge, UK), for $8 \mathrm{hr}$ at $4^{\circ} \mathrm{C}$. After the treatment with primary antibodies the sections were washed three times in PBS for $10 \mathrm{~min}$ and immunostained for $0.5 \mathrm{hr}$ at $20^{\circ} \mathrm{C}$ with $0.1 \mu \mathrm{g} / \mathrm{ml}$ of second antibodies: Alexa Fluor (AF) 488 or 568-conjugated goat anti-hamster and goat anti-rabbit IgGs (Probes Invitrogen Com., Eugene, OR). The immunostained sections were mounted in $50 \%$ polyvinylpyrrolidone solution and examined by fluores- cence microscopy (BZ-8100, Keyence Corp., Osaka, Japan) or laser-scanning microscopy (LSM710, Carl Zeiss, Jena, Germany) with a $\times 63$ oil planapochromatic objective lens (numerical aperture $\times 1.3$ ).

\section{Reverse transcription (RT)-PCR and real-time PCR}

The brain tissue was peeled away from the cerebrum within a $2 \mathrm{~mm}$ square by an 18-gauge needle under a stereoscopic microscope. Total RNA extraction from tissue of the cerebral cortex, hippocampus, thalamus, caudate nucleus, and corpus callosum was performed with a QIAshredder column and RNeasy kit (Qiagen, Inc., Tokyo, Japan). Contaminating genomic DNA was removed using DNAfree (Ambion, Huntingdon, UK), and the RT was performed on $30 \mathrm{ng}$ of total RNA, followed by 30 cycles of PCR for amplification using the Ex Taq hot start version (Takara Bio Inc., Otsu, Japan) with 50 pM of primer sets: mouse $\beta$-actin (forward, 5'-GTTCTACAAATGTGGCTG AGGA; reverse, 5'-ATTGGTCTCAAGTCAGTGTACAG, $411 \mathrm{bp}$ ), mouse podoplanin coding sequence region (forward, 5'-CACCTCAGCAACCTCAGAC; reverse, 5'-AA GACGCCAACTATGATTCCAA, 192 bp), where the specificities had been confirmed by the manufacturer (Sigma-Genosys Ltd., Cambridge, UK). The RT-PCR products were separated on $2 \%$ agarose gel (NuSieve; FMC, Rockland, ME, USA) and visualized by Syber Green (Takara). The correct size of the amplified PCR products was confirmed by gel electrophoresis and amplification of accurate targets was confirmed by sequence analysis. To quantify podoplanin mRNA generation, cDNA samples were analyzed by real-time quantitative PCR. A total of $1 \mu \mathrm{l}$ of cDNA was amplified in a $25-\mu 1$ volume of PowerSYBR Green PCR Master Mix (Applied Biosystems, Foster City, CA, USA) in a Stratagene Mx3000P real-time PCR system (Agilent Technologies, Inc., Santa Clara, CA, USA), and the fluorescence was monitored at each cycle. Cycle parameters were $95^{\circ} \mathrm{C}$ for $15 \mathrm{~min}$ to activate Taq followed by 35 cycles of $95^{\circ} \mathrm{C}$ for $15 \mathrm{~s}, 58^{\circ} \mathrm{C}$ for $1 \mathrm{~min}$, and $72^{\circ} \mathrm{C}$ for 1 min. For the real-time analysis, two standard curves were constructed from amplicons for both the $\beta$-actin and target genes (podoplanin) in three serial 4-fold dilutions of cDNA. The $\beta$-actin or target gene cDNA levels in each sample were quantified against $\beta$-actin or the target gene standard curves by allowing the Mx3000P software to accurately determine each cDNA unit. Finally, target gene cDNA units in each sample were normalized to $\beta$-actin cDNA units. Thus, the relative target gene expression units were expressed as arbitrary units, calculated according to the following formula: relative target gene expression units=target gene cDNA units/ $\beta$-actin cDNA units.

\section{Measurement of the number of podoplanin and GFAP positive cells}

The numbers of podoplanin and GFAP double positive glial cells were determined by counting at five different areas $(75 \mu \mathrm{m} \times 75 \mu \mathrm{m})$. All experiments were repeated five times, and the data are expressed as means \pm standard devi- 
ations (SD). Statistically significant differences $(\mathrm{p}<0.05)$ were determined by the unpaired two-tailed Student's $t$ test with STATVIEW 4.51 software (Abacus concepts, Calabasas, CA).

\section{Measurements of the areas of podoplanin expressing cells}

The areas of podoplanin-positive glial cells were measured at ten different spots $(0.36 \mathrm{~mm} \times 0.36 \mathrm{~mm})$ in the mouse brain section images at $100 \times$ magnification using Image J (National Institutes of Health, Bethesda, MD). All experiments were repeated five times, and data are expressed as means \pm SD. Statistically significant differences $(p<0.05)$ were determined by the unpaired two-tailed Student's t test with STATVIEW 4.51 software (Abacus).

\section{Statistics}

Real-time PCR analysis was repeated five times and the data was expressed as mean $\pm \mathrm{SD}$. The statistical significance of differences $(\mathrm{p}<0.05)$ was determined by the Student's $t$ test and one-way ANOVA with STATVIEW 4.51 software (Abacus concepts, Calabasas, CA, USA).

\section{Results}

The immunohistochemical examination of the mouse tongue showed that the lingual muscle and blood vessels are podoplanin-negative but that the lymphatic vessels and perineurium around the lingual nerve are podoplaninpositive (Fig. 1). The immunohistochemical examination of the cranial bone showed that the periosteum, osteocytes, dura mater, arachnoid, and pia mater are also podoplaninpositive (Fig. 1). The immunohistochemical examination on the mouse spinal cord showed that the meninges, satellite cells in the dorsal root ganglion, periosteum of vertebra, glia cells around ventral horn, and schwann cells in the ventral and dorsal roots are podoplanin-positive (Fig. 2). The immunohistochemical study of the mouse eye showed that the optic nerve, pigment epithelium, retina near the disk, chorioidea, sclera, iris, lens epithelium, corneal epithelium, and conjunctival epithelium are podoplanin-positive (Fig. 3). For the mouse brain the immunohistochemical study showed that the choroid plexus and ependymal cells are podoplanin-positive, and that in the brain parenchyma there are cells expressing both podoplanin and the astrocyte marker GFAP in the cerebral cortex, hippocampus, thala-
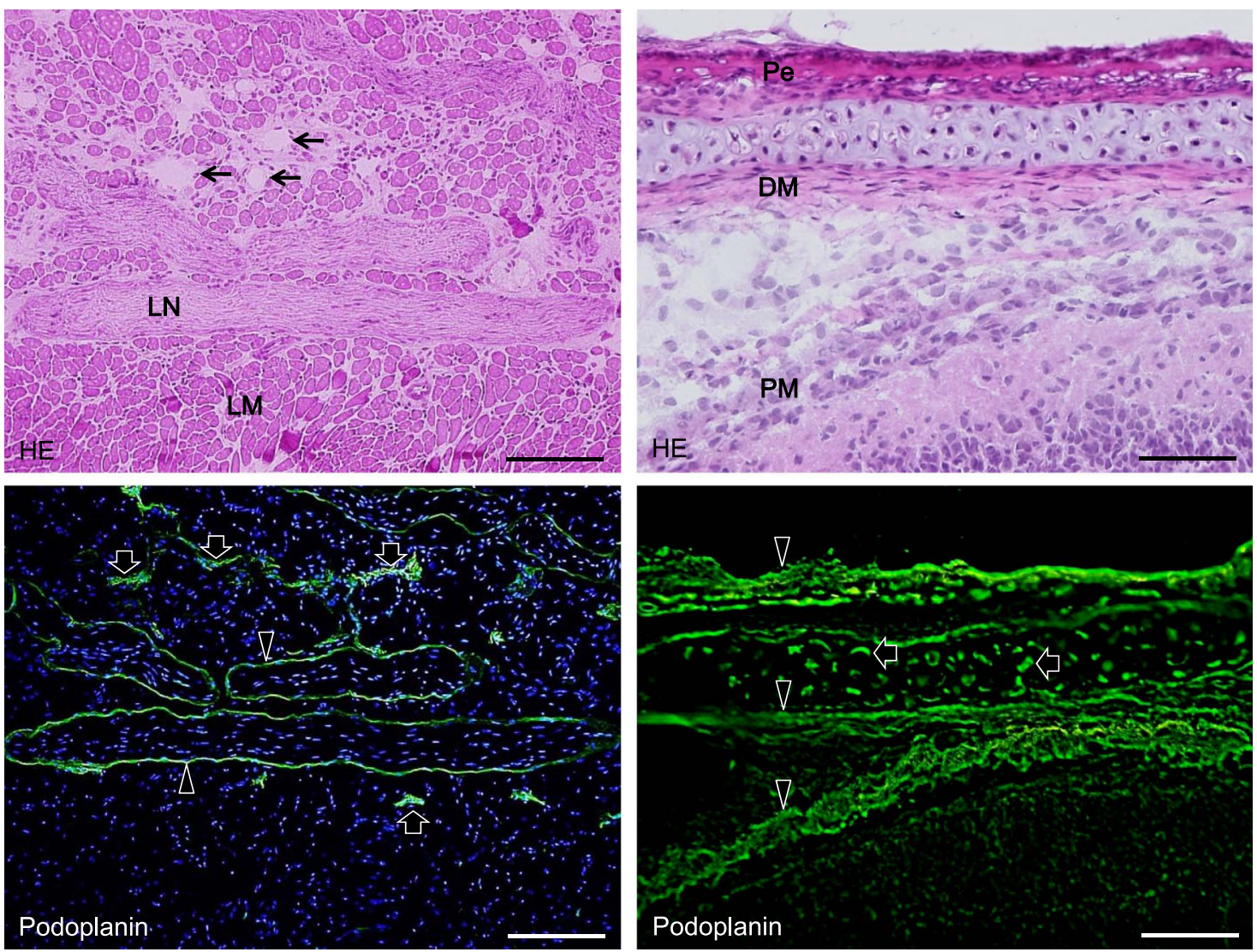

Fig. 1. Podoplanin expression in mouse lymphatic vessels, bone, and connective tissue. The immunostained sections were re-stained by H-E staining. In the mouse tongue, the lingual muscle and blood vessels (arrows in HE) are podoplanin-negative but lymphatic vessels (arrows in immunostaining) and the perineurium around the lingual nerve (arrowheads) are podoplanin-positive. In the cranium, podoplanin-positive areas (arrowheads) are observed in periosteum (Pe), dura mater (DM), and pia mater (PM). Osteocytes in the cranial bone are also podoplaninpositive (arrows). Bar $=100 \mu \mathrm{m}$. 

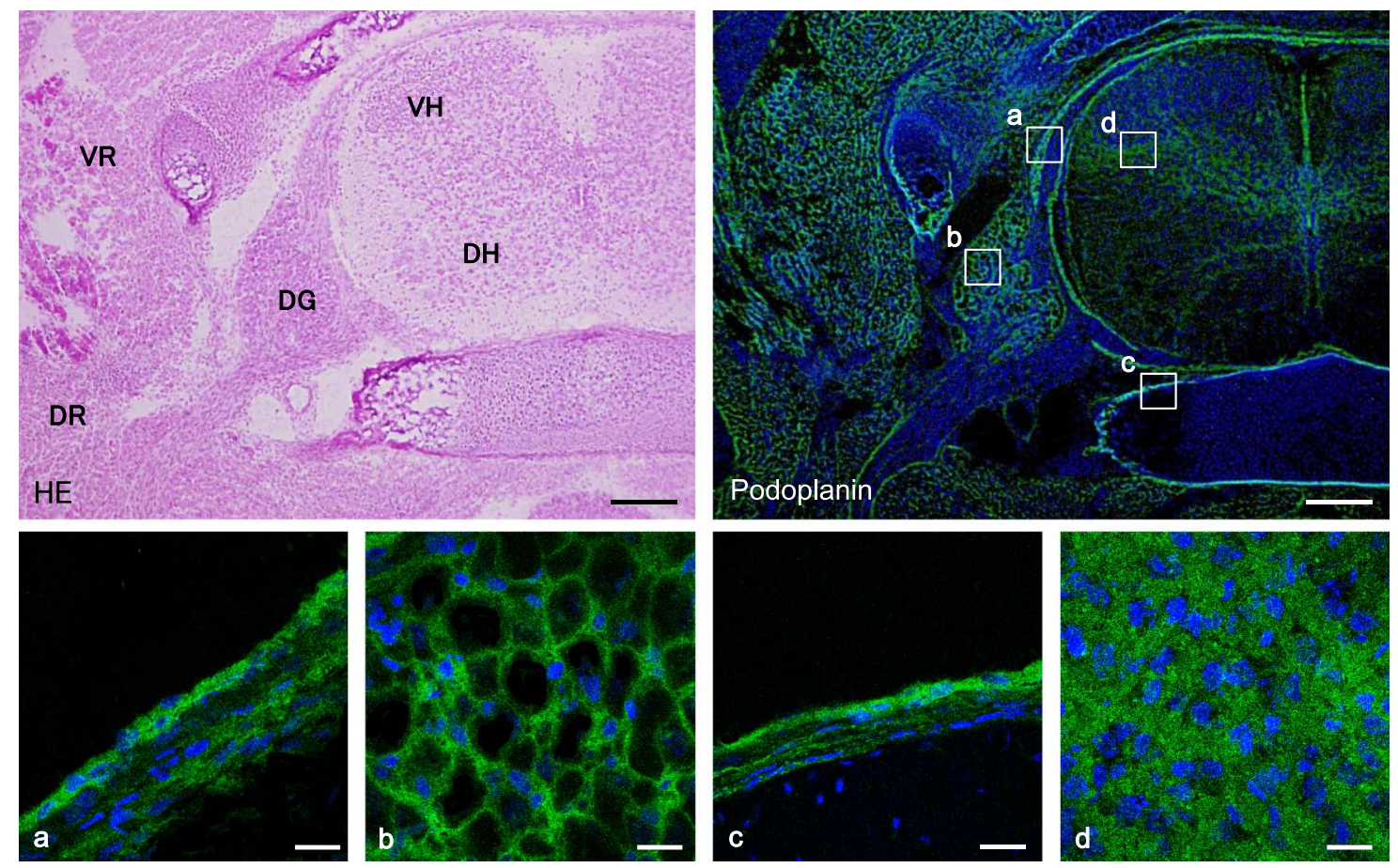

Fig. 2. Immunostaining for podoplanin in a horizontal section of mouse spinal cord. The immunostained sections were re-stained by H-E staining. The regions which are highlighted by boxes in the top right podoplanin immunostaining panel correspond to the lower panels with laser-scanning microscopic images (a-d). In the spinal cord, the podoplanin-positive areas are observed in (a) meninges, (b) satellite cells of the dorsal root ganglion (DG), (c) periosteum of the vertebra, and (d) glia cells of the ventral horn (VH). Schwann cells in the ventral root (VR) and in the dorsal root (DR) are also podoplanin-positive. Bar=100 $\mu \mathrm{m}$ (upper), $20 \mu \mathrm{m}$ (lower).
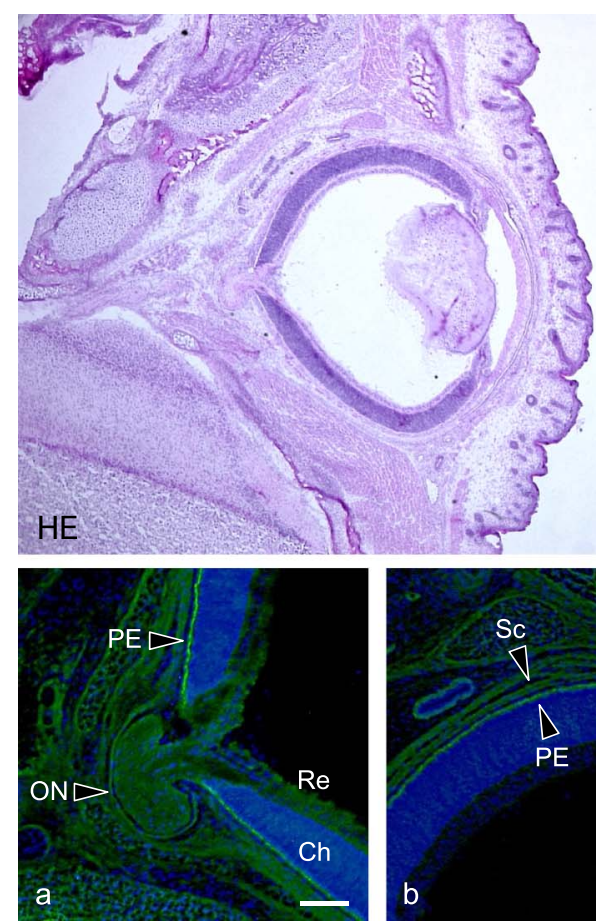
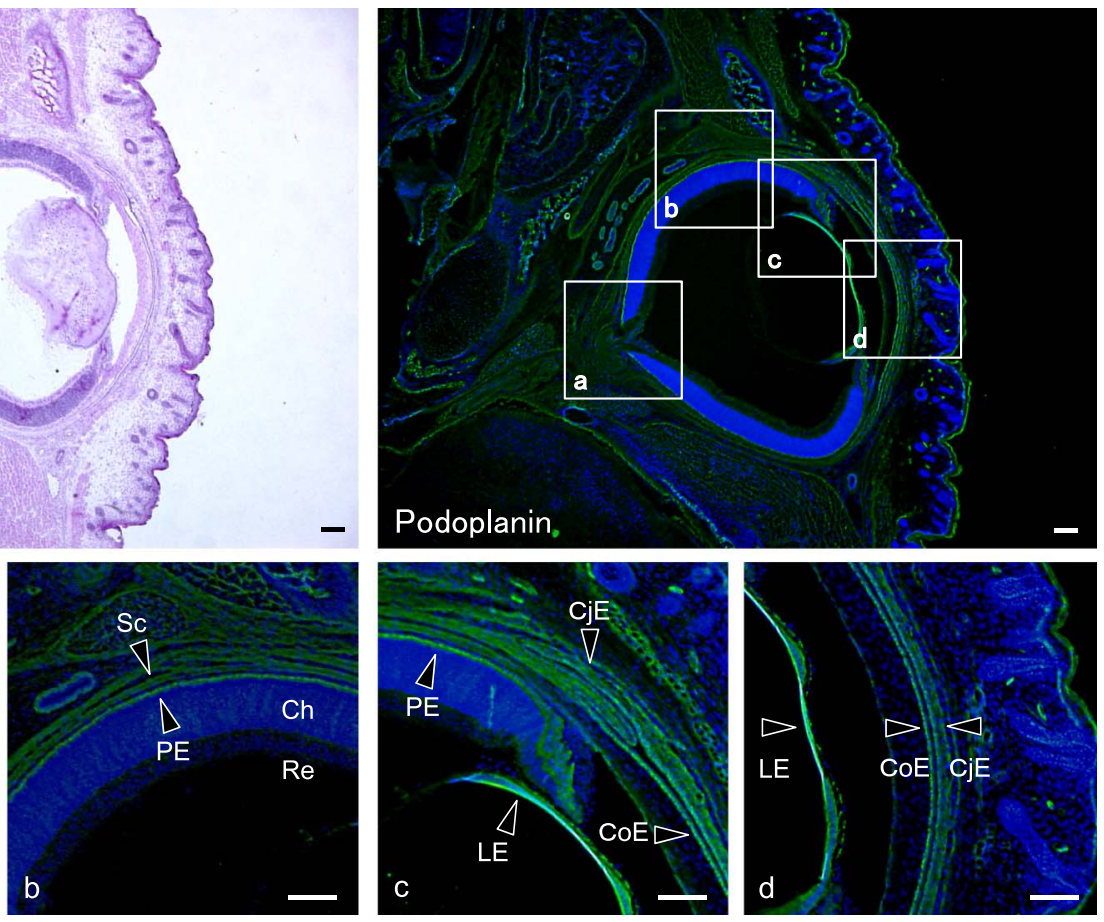

Fig. 3. Immunostaining for podoplanin in a horizontal section of the mouse eye. The immunostained sections were re-stained by H-E staining The regions which are highlighted by boxes in the upper figure of podoplanin immunostaining correspond to the lower figures of laser-scanning microscopic images (a-d). The podoplanin-positive areas are the optic nerve (ON), pigment epithelium (PE), retina (Re) near the optic disk, chorioidea $(\mathrm{Ch})$, sclera $(\mathrm{Sc})$, lens epithelium (LE), corneal epithelium (CoE), and conjunctival epithelium (CjE). Bar=100 $\mu \mathrm{m}$. 

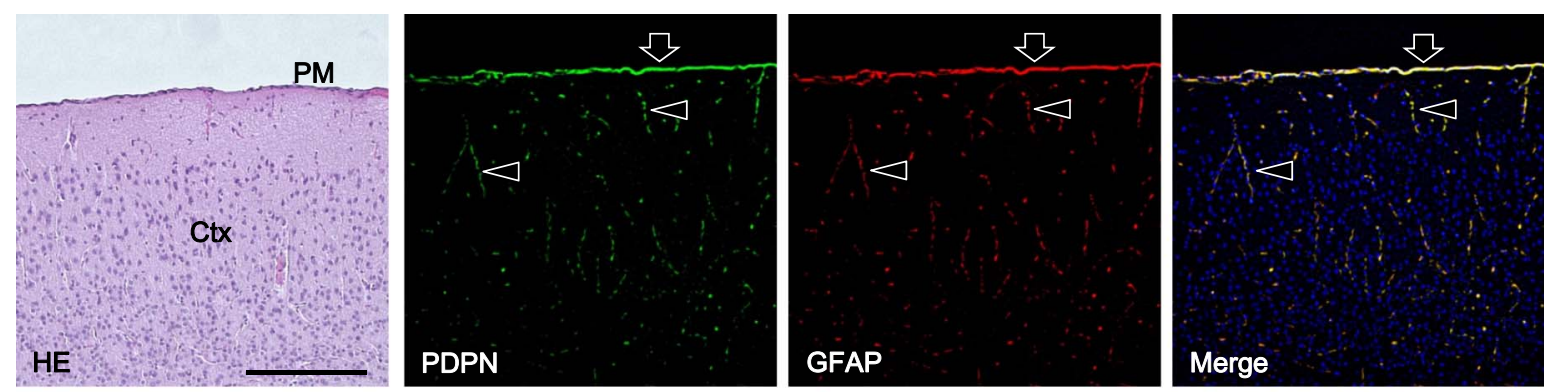

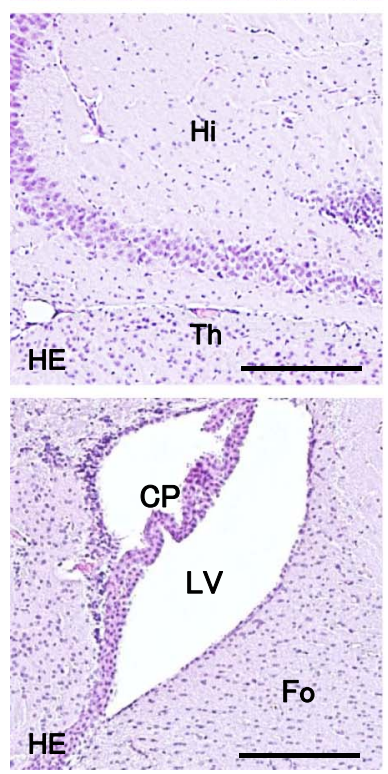

HE
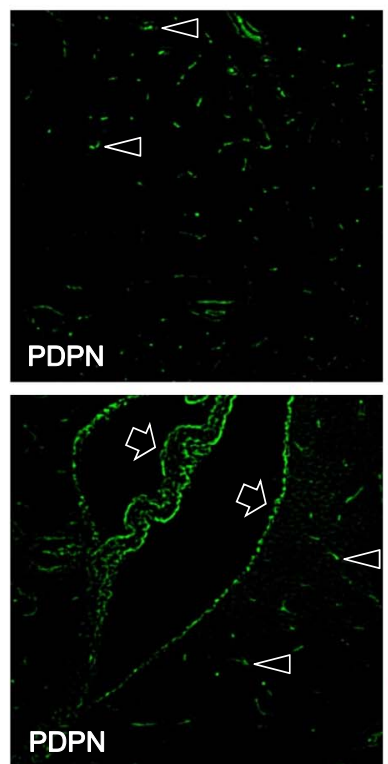

PDPN
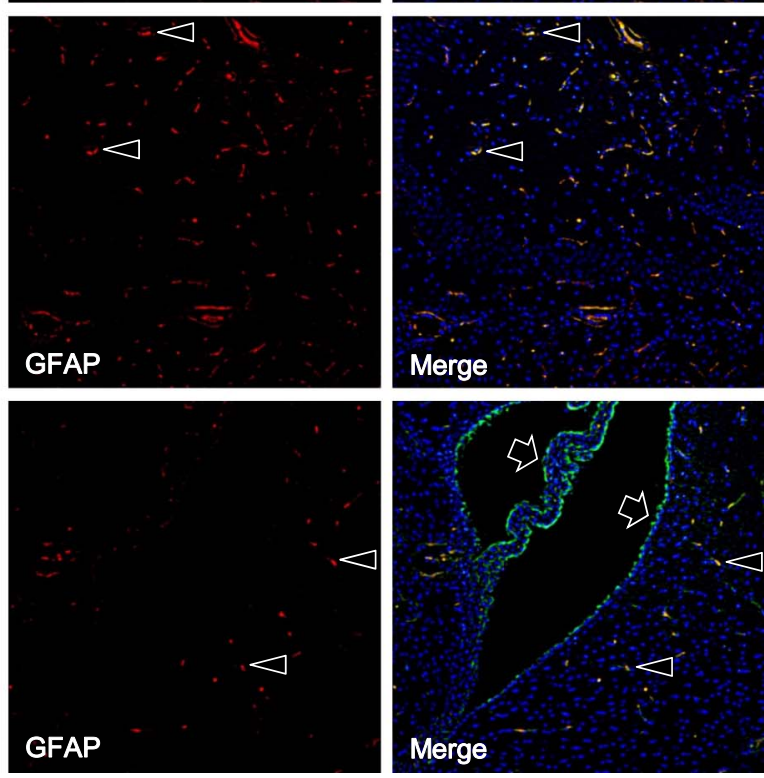

GFAP

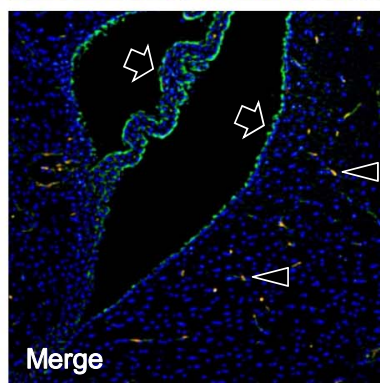

Merge

Fig. 4. Immunostaining for podoplanin in a horizontal section of the mouse brain. The immunostained sections were re-stained by H-E staining. There are cells expressing both podoplanin and GFAP (arrowheads) in the cerebral cortex (Ctx), hippocampus (Hi), thalamus (Th), and fornix (Fo). Pia mater (PM), choroid plexus (CP), and ependymal cells in the lateral ventricle are also podoplanin- and GFAP-double positive (arrows). Bar $=100 \mu \mathrm{m}$.

mus, and fornix (Fig. 4). In the quantitative analysis for the podoplanin and GFAP double positive cells in the mouse brain, there were no differences in areas and numbers of double positive cells in the cerebral cortex, hippocampus, thalamus, and caudate nucleus (Fig. 5). These areas and cell numbers were statistically significantly larger than the numbers in the fornix. In the analysis for the gene expression of podoplanin in the mouse brain, there were no differences in the mRNA amounts in the cerebral cortex, hippocampus, thalamus, and caudate nucleus but these mRNA amounts were statistically significantly larger than those in the fornix (Fig. 6).

\section{Discussion}

The study used anti-podoplanin where the specificity has been confirmed in our previous studies [30], and showed that the reaction products were observed in lymphatic vessels but not in blood vessels as also reported elsewhere, showing that the immunohistochemistry for podoplanin was successful (Fig. 1). The immunohisto- chemical examination on the cranial and vertebra bone showed that osteocytes, periosteal cells, and dura mater fibroblasts were podoplanin-positive (Figs. 1, 2). It is thought that the cranial and vertebra bone-related mesenchymal cells have the ability to produce podoplanin [31]. In the peripheral nerve tumors, it has been reported that podoplanin expresses in $76-100 \%$ of schwannomas and in $21 \%$ of spindle malignant peripheral nerve sheath tumors [10]. The study here showed that perineurial fibroblasts of peripheral nerves, satellite cells in the ganglion, glia cells and schwann cells in the spinal cord and nerve roots, are generally podoplanin-positive (Figs. 1, 2). This suggests that cells supporting nerves like glia cells have the ability to express podoplanin. It has recently been reported that the expression of podoplanin is a characteristic of meningiomas which arise from arachnoid mater covering the surface of the brain and spinal cord [28]. The study here showed that meningeal fibroblasts and glial cells express podoplanin (Figs. 1, 2), suggesting that the expression of podoplanin is a common characteristic among cells supporting meninges. In the mouse eye podoplanin expression was observed in 
(A)

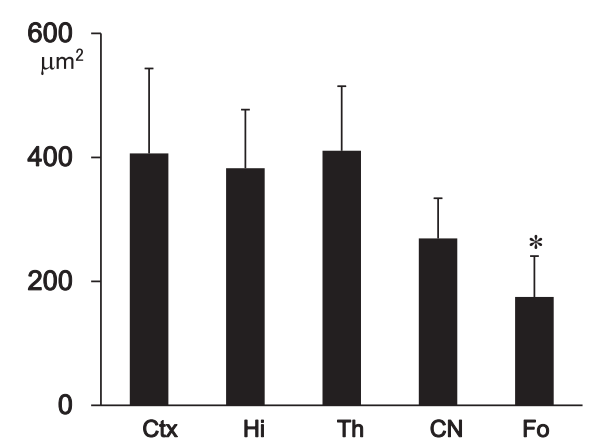

(B)

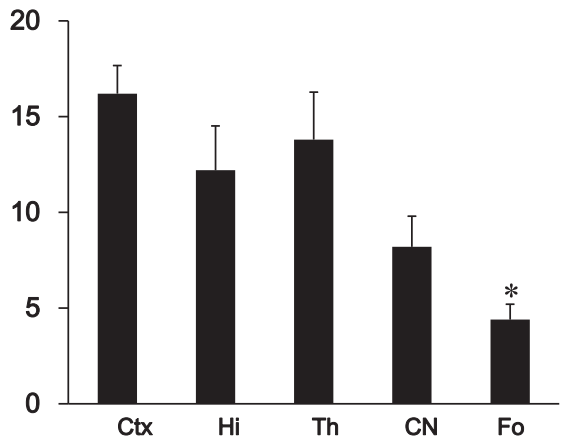

Fig. 5. Quantitative analysis of podoplanin-positive cells in the mouse brain. (A) Area of podoplanin and GFAP double positive cells in the cerebral cortex (Ctx), hippocampus (Hi), thalamus (Th), caudate nucleus (CN), and fornix (Fo). The areas of podoplanin-positive cells in the fornix were statistically significantly smaller than other areas. There were no differences in the tested areas except for the fornix. Data are expressed as means \pm SD. * Significantly different $(\mathrm{p}<0.01)$. (B) Number of podoplanin and GFAP double positive cells in the cerebral cortex (Ctx), hippocampus (Hi), thalamus (Th), caudate nucleus $(\mathrm{CN})$, and fornix (Fo). Average of the number of cells in five spots $\left(75 \mu \mathrm{m}^{2} \times 75 \mu \mathrm{m}^{2}\right)$ in the fornix were statistically significantly fewer than in other areas. There were no differences in the tested areas except for in the fornix. Data are expressed as means \pm SD. $*$ Significantly different $(\mathrm{p}<0.01)$.

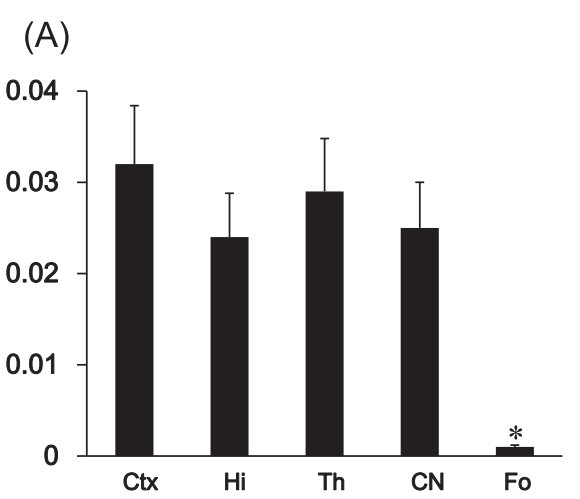

(B)

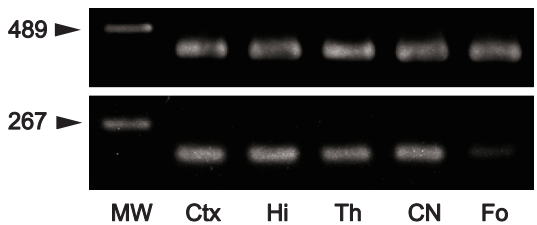

Fig. 6. Gene analysis for the expression of podoplanin mRNA in the mouse brain. Total RNA extraction from tissue of the cerebral cortex (Ctx), hippocampus (Hi), thalamus (Th), caudate nucleus $(\mathrm{CN})$, and fornix (Fo) were examined. (A) Real-time PCR analysis. Relative mRNA amounts are expressed in arbitrary units. Target gene cDNA units in each sample were normalized to $\beta$-actin cDNA units. The podoplanin mRNA amount in the fornix was significantly smaller than in other areas. There were no differences in the tested areas except for in the fornix. *Significantly different $(\mathrm{p}<0.01)$. (B) RT-PCR analysis. The intensity of amplicon for podoplanin mRNA was significantly lower in the fornix than in other areas. There were no differences in the tested areas except for in the fornix. The intensity of amplicon for $\beta$-actin mRNA was similar among all areas. epithelial cells including pigment, lens, cornea, iris, and conjunctiva, and also in fibroblasts of the chorioidea (Fig. 3), suggesting that the expression of podoplanin is a common characteristic of the epithelial and endothelial cells which form the eyes.

There have been reports that podoplanin is expressed in $25.6 \%$ of anaplastic astrocytomas and in $47.0 \%$ of glioblastomas, and that podoplanin mRNA and protein expression are markedly higher in glioblastomas than in anaplastic astrocytomas [19]. The podoplanin expression may be seen as a useful index of the malignancy of glioma $[12,13,27]$, however, this study showed that there are podoplanin-positive cells in the choroid plexus and ependymal cells as also reported previously [11], and further, that in the mouse brain parenchyma there are cells expressing podoplanin and GFAP which is an intermediate filament specific for astrocytes (Fig. 4). There were no differences in the podoplanin and GFAP double positive cell distributions of the several portions in the brain parenchyma except in the fornix (Figs. 5, 6), suggesting that the expression of podoplanin is a characteristic of astrocytes.

It has been reported that a biological role of podoplanin is the rearrangement of the actin cytoskeleton and dependent on the binding activity with a cytoplasmic linker protein ezrin. Podoplanin up-regulates a Rho-GTPase activity resulting in ezrin phosphorylation [17, 26, 34]. Phosphorylated ezrin mediates the connection between membrane proteins and $\mathrm{F}$-actin, and an increase in podoplanin at the cell membrane promotes the formation of membrane-actin structures critical for the cell shape and also induces plasma membrane extensions based on the cytoskeleton rearrangement $[2,5,15,27]$. Podoplanin may contribute to form the cytoskeleton of the peripheral and central nervous system support cells, and of eye constituents. 


\section{References}

1. Amano, I., Imaizumi, Y., Kaji, C., Kojima, H. and Sawa, Y. (2011) Expression of podoplanin and classical cadherins in salivary gland epithelial cells of klotho-deficient mice. Acta Histochem. Cytochem. 44; 267-276.

2. Belkina, N. V., Liu, Y., Hao, J. J., Karasuyama, H. and Shaw, S. (2009) LOK is a major ERM kinase in resting lymphocytes and regulates cytoskeletal rearrangement through ERM phosphorylation. Proc. Natl. Acad. Sci. US A 106; 4707-4712.

3. Breiteneder-Geleff, S., Matsui, K., Soleiman, A., Meraner, P., Poczewski, H., Kalt, R., Schaffner, G. and Kerjaschki, D. (1997) Podoplanin, novel43-kd membrane protein of glomerular epithelial cells, is down-regulated in puromycin nephrosis. Am. J. Pathol. 151; 1141-1152.

4. Chu, A. Y., Litzky, L. A., Pasha, T. L., Acs, G. and Zhang, P. J. (2005) Utility of D2-40, a novel mesothelial marker, in the diagnosis of malignant mesothelioma. Mod. Pathol. 18; 105-110.

5. Gautreau, A., Poullet, P., Louvard, D. and Arpin, M. (1999) Ezrin, a plasma membrane-microfilament linker, signals cell survival through the phosphatidylinositol 3-kinase/Akt pathway. Proc. Natl. Acad. Sci. US A 96; 7300-7305.

6. Hata, M., Ueki, T., Sato, A., Kojima, H. and Sawa, Y. (2008) Expression of podoplanin in the mouse salivary glands. Arch. Oral Biol. 53; 835-841.

7. Hata, M., Amano, I., Tsuruga, E., Kojima, H. and Sawa, Y. (2010) Immunoelectron microscopic study of podoplanin localization in mouse salivary gland myoepithelium. Acta Histochem. Cytochem. 43; 77-82.

8. Imaizumi, Y., Amano, I., Tsuruga, E., Kojima, H. and Sawa, Y. (2010) Immunohistochemical examination for the distribution of podoplanin-expressing cells in developing mouse molar tooth germs. Acta Histochem. Cytochem. 43; 115-121.

9. Ishizawa, K., Komori, T., Shimada, S. and Hirose, T. (2009) Podoplanin is a potential marker for the diagnosis of ependymoma: a comparative study with epithelial membrane antigen (EMA). Clin. Neuropathol. 28; 373-378.

10. Jokinen, C. H., Dadras, S. S., Goldblum, J. R., van de Rijn, M., West, R. B. and Rubin, B. P. (2008) Diagnostic implications of podoplanin expression in peripheral nerve sheath neoplasms. Am. J. Clin. Pathol. 129; 886-893

11. Kaji, C., Tomooka, M., Kato, Y., Kojima, H. and Sawa, Y. (2012) The expression of podoplanin and classic cadherins in the mouse brain. J. Anat. 220; 435-446.

12. Kaneko, M., Kato, Y., Kunita, A., Fujita, N., Tsuruo, T. and Osawa, M. (2004) Functional sialylated O-glycan to platelet aggregation on Aggrus (T1alpha/Podoplanin) molecules expressed in Chinese hamster ovary cells. J. Biol. Chem. 279; 38838-38843

13. Kato, Y., Fujita, N., Kunita, A., Sato, S., Kaneko, M. and Osawa, M. (2003) Molecular identification of Aggrus/T1 $\alpha$ as a platelet aggregation-inducing factor expressed in colorectal tumors. $J$. Biol. Chem. 278; 51599-51605.

14. Kimura, N. and Kimura, I. (2005) Podoplanin as a marker for mesothelioma. Pathol. Int. 55; 83-86.

15. Lan, M., Kojima, T., Murata, M., Osanai, M., Takano, K., Chiba, H. and Sawada, N. (2006) Phosphorylation of ezrin enhances microvillus length via a p38 MAP-kinase pathway in an immortalized mouse hepatic cell line. Exp. Cell Res. 312; 111-120.

16. Marsee, D. K., Pinkus, G. S. and Hornick, J. L. (2009) Podoplanin (D2-40) is a highly effective marker of follicular dendritic cells. Appl. Immunohistochem. Mol. Morphol. 17; 102-107.

17. Martín-Villar, E., Megías, D., Castel, S., Yurrita, M. M., Vilaró, S. and Quintanilla, M. (2006) Podoplanin binds ERM proteins to activate RhoA and promote epithelial-mesenchymal transition. J. Cell Sci. 119; 4541-4553.

18. Mei, Y., Zhang, P., Zuo, H., Clark, D., Xia, R., Li, J., Liu, Z. and Mao, L. (2013) Ebpl activates podoplanin expression and contributes to oral tumorigenesis. Oncogene doi: 10.1038/onc. 2013.354 (in print).
19. Mishima, K., Kato, Y., Kaneko, M. K., Nishikawa, R., Hirose, T. and Matsutani, M. (2006) Increased expression of podoplanin in malignant astrocytic tumors as a novel molecular marker of malignant progression. Acta Neuropathol. 111; 483-488.

20. Noda, Y., Amano, I., Hata, M., Kojima, H. and Sawa, Y. (2010) Immunohistochemical examination of the distribution of cells expressed lymphatic endothelial marker podoplanin and LYVE-1 in the mouse tongue tissue. Acta Histochem. Cytochem. 43; 61-68.

21. Peterziel, H., Muller, J., Danner, A., Barbus, S., Liu, H. K., Radlwimmer, B., Pietsch, T., Lichter, P., Schutz, G., Hess, J. and Angel, P. (2012) Expression of podoplanin in human astrocytic brain tumors is controlled by the PI3K-AKT-AP-1 signaling pathway and promoter methylation. Neuro. Oncol. 14; 426-439.

22. Ramirez, M. I., Millien, G., Hinds, A., Cao, Y. X., Seldin, D. C. and Williams, M. C. (2003) T1alpha, a lung type I cell differentiation gene, is required for normal lung cell proliferation and alveolous formation at birth. Dev. Biol. 256; 61-72.

23. Sawa, Y., Iwasawa, K. and Ishikawa, H. (2008) Expression of podoplanin in the mouse tooth germ and apical bud cells. Acta Histochem. Cytochem. 41; 121-126.

24. Schacht, V., Ramirez, M. I., Hong, Y. K., Hirakawa, S., Feng, D., Harvey, N., Williams, M., Dvorak, A. M., Dvorak, H. F., Oliver, G. and Detmar, M. (2003) T1a/podoplanin deficiency disrupts normal lymphatic vasculature formation and causes lymphedema. EMBO J. 22; 3546-3556.

25. Schacht, V., Dadras, S. S., Johnson, L. A., Jackson, D. G., Hong, Y. K. and Detmar, M. (2005) Up-regulation of the lymphatic marker podoplanin, a mucin-type transmembrane glycoprotein, in human squamous cell carcinomas and germ cell tumors. Am. J. Pathol. 166; 913-921

26. Scholl, F. G., Gamallo, C., Vilar, S. and Quintanilla, M. (1999) Identification of PA2.26 antigen as a novel cell-surface mucintype glycoprotein that induces plasma membrane extensions and increased motility in keratinocytes. J. Cell Sci. 112; 4601-4613.

27. Shibahara, J., Kashima, T., Kikuchi, Y., Kunita, A. and Fukayama, M. (2006) Podoplanin is expressed in subsets of tumors of the central nervous system. Virchows Arch. 448; 493-499.

28. Shintaku, M., Honda, T. and Sakai, T. (2010) Expression of podoplanin and calretinin in meningioma: an immunohistochemical study. Brain Tumor Pathol. 27; 23-27.

29. Sonne, S. B., Herlihy, A. S., Hoei-Hansen, C. E., Nielsen, J. E., Almstrup, K., Skakkebaek, N. E., Marks, A., Leffers, H. and Rajpert-De Meyts, E. (2006) Identity of M2A (D2-40) antigen and gp36 (Aggrus, T1A-2, podoplanin) in human developing testis, testicular carcinoma in situ and germ-cell tumours. Virchows Arch. 449; 200-206.

30. Takata, S., Sawa, Y., Uchiyama, T. and Ishikawa, H. (2013) Expression of toll-like receptor 4 in glomerular endothelial cells under diabetic conditions. Acta Histochem. Cytochem. 46; 35-42.

31. Wetterwald, A., Hoffstetter, W., Cecchini, M. G., Lanske, B., Wagner, C., Fleisch, H. and Atkinson, M. (1996) Characterization and cloning of the E11 antigen, a marker expressed by rat osteoblasts and osteocytes. Bone 18; 125-132.

32. Williams, M. C., Cao, Y., Hinds, A., Rishi, A. K. and Wetterwald, A. (1996) Tlalpha protein is developmentally regulated and expressed by alveolar type I cells, choroid plexus and ciliary epithelia of adult rats. Am. J. Respir. Cell Mol. Biol. 14; 577-585.

33. Yu, H., Gibson, J. A., Pinkus, G. S. and Hornick, J. L. (2007) Podoplanin (D2-40) is a novel marker for follicular dendritic cell tumors. Am. J. Clin. Pathol. 128; 776-782.

34. Yu, Y., Khan, J., Khanna, C., Helman, L., Meltzer, P. S. and Merlino, G. (2004) Expression profiling identifies the cytoskeletal organizer ezrin and the developmental homeoprotein Six-1 as key metastatic regulators. Nat. Med. 10; 175-181.

This is an open access article distributed under the Creative Commons Attribution License, which permits unrestricted use, distribution, and reproduction in any medium, provided the original work is properly cited. 